\title{
Dynamics Layer of the Sliding Contact Collector Elements
}

\author{
S.A. Romanishina, D.Yu. Katyuk \\ Dept. processes, machinery and agricultural equipment \\ Zhytomyr National Agroecological University, ZhNAEU \\ Zhytomyr, Ukraina \\ sar62@yandex.ua
}

\author{
V.S. Deeva, S.M. Slobodyan \\ Institute of Natural Resources \\ Tomsk Polytechnic University, TPU \\ Tomsk, Russia \\ veradee@mail.ru,slobodyan_sm@ukr.nct
}

\begin{abstract}
The problems of the dynanic interaction of the collector elements are covered. It takes into account the physical properties of the material and behavior of their parameters and the features of mechanical coupling. A mathematical model of dynamics of fractions of random destruction in the contact layer conductivity is created. II creates for sliding contact elements of collector in steady-state.
\end{abstract}

Keywords - haos; sliding contact; destruction; wear; dynamics; layer; model.

\section{INTRODUCTION}

Dynamic contact interaction of material solids and fields because of the forces and friction actions leading to destruction is multifactor. Multifactorial destruction of contacting surface takes into account the fraction separating and fraction moving away from space [1-14].

Solving problems of wear and tear (including non-ferrous metals) is connected with the creation of effective methods to reduce the destruction of the collector elements. It concerns all fields of science and technology, particularly electrical engineering [15-24]. The major condition for creating of effective technology to prevent wear of sliding contact elements is clear understanding of destruction process of interaction of such elements.

\section{THE URGENCY OF THE TASK}

More complete (integrated) study and evaluation of the influence of wear leads to the need for a more general description of the process of dynamic contact interaction of solids. There is over the variety of conditions and results of wear. The wear is by changes of the properties of solids material in contact space (CS) of their dynamic interaction. Contact interaction process of sliding solids generates a random variety of CS as a sliding layer (SL). The peculiarity of this SL of dynamic contacting solids dynamic interaction is available of fractions into SL. These fractions are generated by grains of contacting solids destruction and atmospheric environment. These fractions are of different density, size, elasticity, and other properties. Such multiphase peculiarity of SL of dynamic contacting solids takes place practically in many types of frictional interaction of contact elements. A generalized analysis of the properties of the SL allows considering the combined effect of all destruction factors (adhesion, fatigue, etc.) as a dynamically property changing's of SL in real time under the assumed conditions.

In the view of the authors, such study to solve problems of diagnosis, prediction and control of the destruction of contact elements in real time is a necessary, appropriate and relevant.

Prediction of the elements state depends on the accuracy of a physical model of contact interaction or measurement parameters during diagnosis of their situation [1-5,16-23] and their methods [6-15,24-27]. This prediction is a basic of technical products and theirs components operation.

Known methods of assessment of sliding contact elements [6-15] not fully capture the physics of random phenomena in the space of a thin SL of the solids dynamic interaction.

\section{FORMULATION OF RF.SEARCH}

Because of article limitation we don't pretend on exhaustive characterization CS studies, but we can formulate a general idea about the sliding contact elements features. Using the general principle studies of the physical processes in systems of densely packed discrete structure, we take a dynamic model $[6-15]$, which takes into account changes in the contact interaction of sliding solids as the basis of the model of the destruction process of the solid sliding on the surface of another solid. Conditions of sliding solids interaction vary randomly, which leads to random physical, mechanical and other processes in the SL under the influence of environmental and other factors.

Despite the many of studies [1-5] on solids fritting, the random nature of contact elements has received and despitc the many of studies [6-15] concerning the destruction of the different contact surface of solids there are a few publications about the dynamics and kinetics of the space between two sliding solids. The space of sophisticated stochastic interaction of collector elements is beyond the comprehension. Therefore such researches are of interest of estimation of sliding contact elements viability. This is actually to electric machine industry and physics of sliding interaction of condensed substance.

The analysis of thin layer of destructing of collector elements in space limited by area of sliding element in steady state is carried out at work.

Look over the physical process of sliding contact elements wear. Many of studies [1-15] show that fractions of sliding 
2015 IEEE 35th International Conference on Electronics and Nanotechnology (ELNANO)

solid are transferred to the rotating solid and they are deposited thereon in the form of separate islets and scratch but not in the form of continuous pellicle. Wear caused by a mechanical impact on the contacting surface. This conclusion is strongly supported by experiments [12-13, 16-27].

\section{IV.DYNAMICS OF THE CONTACT LAYER STRUCTURE}

Velocity of changing of the contact set is determined by the dynamics of emission of destructing elements.

We'll take that the contact area of sliding elements in steady state is constant (Fig. 1). Example: bank wiper sliding over the surface of electrical machine slip-ring (commutator) has the constant rotor angular rotating velocity.

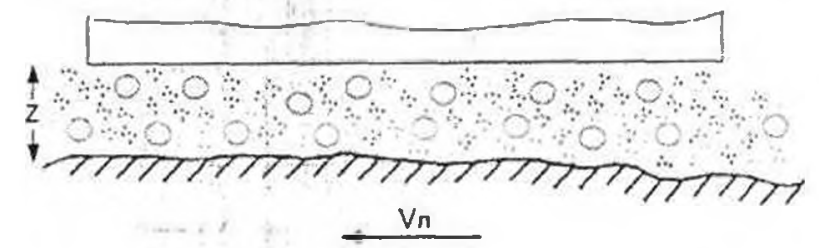

Fig. 1. Formation of contact area of sliding elements ( $z$ - high of CS, $\mathrm{V}_{\mathrm{n}}$ - linear rate of rotation movement of surface of element)

Possible destruction of the lateral surfaces of the contact element (bank wiper) can be neglected because of the insignificance of this factor. This destruction is formed during the motion of bank wiper under pressure of the spring member inside the brush holder. Then specific volume of fractions destruction of the SL of bank wiper (we take bank wiper as condensed substance of packed compound) through the crosssection of brush holder (canal of fraction flow movement as a result of tribological destruction) in unit time is equal

$$
: V_{f r}=s_{f r} f_{f r} t,
$$

where $s_{f r}$ is a square of canal cross-section, $\nu_{f r}$ is an average linear rate of fraction movement along canal (longitudinal).

Flow velocity of tribological factions in $\mathrm{CS}$ under the dynamics of interaction of sliding elements of currentcollecting in the coordinate system $(x, y, z)$ is determined by its velocity variations $y_{f r}(x, y, z, t)$. Flow components change in along the coordinate axes:

$$
\begin{aligned}
& v_{\mathrm{x}}(\mathrm{t})=\left[x_{i}(t+\Delta t)-x_{i}(t)\right] / \Delta t ; \\
& v_{\mathrm{y}}(t)=\left[y_{j}(t+\Delta t)-y_{j}(t)\right] / \Delta t ; \\
& v_{z}(t)=\left[z_{i}(t+\Delta t)-z_{i}(t)\right] / \Delta t,
\end{aligned}
$$

where $(x, y, z)$ are the variables in the coordinates system of the contact area and $\Delta t$ is the time slice of the flow fractions rate.

The process of destruction of the solid into fractions is a long-continued. Time of complete destruction of the solid is equal ratio of the solid volume to fractions rate of occurrence and fractions size:

$$
{ }_{p m}=V_{f r} / \bar{n}_{f r} \bar{V}_{i f r}
$$

The fractions generate by destruction of the contact layer of interaction of sliding elements of current-collecting.

In the general case:

$$
\bar{V}_{f r}=\iiint_{(x, y, z)} V_{i f r} d x d y d z=\iiint_{\left(V^{\prime}\right)} V_{i f r} d V .
$$

It is clear that the average number of fractions destruction of sliding elements $\left(\bar{n}_{f r}\right)$ and the fraction size are random interconnected correlated variables. Hence average number of fractions destruction of sliding elements is: $\bar{n} f r=V f r / \bar{V}_{f r}$.

Thus, volume of fractions destruction of sliding elements, going through cross-section of the contact layer is equal the product of cubic content of fractions to the solid density

$$
m_{\rho r}(t)=\rho_{s s} s_{f r} v_{f r} r=\sum_{i=1}^{k} \bar{V}_{i f r}{ }_{f r} \rho i f r .
$$

Here and below we assume that $\rho_{s s} \equiv \rho_{i f f}$, that is the solid density is identical to volume of fractions destruction of sliding elements in smooth space. Although this identity could not be hold, for example, diamond cutter slides along the plane of glass, that is if a solid more elastic than the sliding. Lets define that an average density of tribological flow of fractions destruction of non-uniform solids in CS is the ratio of solid weight to its volume: $\rho_{s}=m i \mathrm{~V}$.

In the general case, the parameters $v_{i f r}, \bar{V}_{i f r}, \rho_{i f r}$ are not constant, that is they depend on time and coordinate. It could leads to violation of the constancy of the density distribution of the fractions across the flow. But taking into account that the problem of research is creation of a model flow of fractions destruction in the steady-state, we consider that $v_{i f r}, \bar{V}_{i f r}, \rho_{i f r}$ are the average centered values $(<\ldots>)$ of characteristics of the destruction process.

Condition of random destruction of the flow fractions stationary supposes the constancy of the average characteristics of flow fractions in all cross-sections of the space. In other words this condition leads to the equation of continuity of flow fractions destruction:

$$
m_{f r}(t)=\rho_{s s} s_{f,} \cdot v_{f r} \cdot=\sum_{i=1}^{k} \bar{V}_{i f r} \cdot v_{f r} \rho_{i f r}=\mathrm{cons} s .
$$

This equation is valid to any type of stationary flow fractions destruction, having no inflow or outflow fractions in CS. We take the model of uniform SL structure in CS with varying specific density. It takes according to moving dynamics, the changing of fractions destruction properties and other factors in the SC. To solve this system, reduce it to ordinary differential equations with separable variables. Consider the steady fractions flow traffic in CS on isothermal 


\section{IEEE 35th International Conference on Electronics and Nanotechnology (ELNANO)}

conditions, i.e. at a constant temperature of SL. Take into account the SL deviation from horizontal by dependence of gravity on the angle $\alpha_{\perp}$ (deviation angle from the horizontal):

$$
-\rho \eta_{p} R_{p} T v^{-2} d\left(v^{2}\right)+\alpha_{T} d\left(v^{2}\right)+\left\lfloor 2 g \cos \alpha_{\perp}+R_{v} v^{2} / \ell_{e}\right) d z=0(7)
$$

At horizontal layer: $\alpha_{\perp}=0\left(\cos \alpha_{\perp}=1\right)$. At vertical layer: $\alpha_{\perp}=90^{\circ}\left(\cos \alpha_{1}=0\right)$. Gravity directs the fractions flow along layer. The fractions flow fall down and $2 g d z \equiv 0$. Resulting in the equation similar terms:

$$
\frac{\eta_{\mathrm{p}} R_{\mathrm{p}} T d\left(v^{2}\right)}{v^{2}\left[2 g \cos \alpha_{\perp}+R_{v} v^{2} / \ell_{\mathrm{e}}\right]}-\frac{\alpha_{T} d\left(v^{2}\right)}{\left[2 g \cos \alpha_{\perp}+R_{v} v^{2} / \ell_{\mathrm{e}}\right]}=d z
$$

Found patterns of change in the calculation of the coefficients in solving the system of equations: coefficient $a$, depending only on the orientation CS $a=\left(2 g \cos \alpha_{\perp}\right) / \eta_{p} R_{p} T$

and $b=1+\frac{\alpha_{T} \ell_{e}}{R_{v} \ell_{z}} \ln \frac{2 \ell_{e} g \cos \alpha_{\perp}+R_{v} \ell_{z} v_{\ell}^{2}}{2 \ell_{e} g \cos \alpha_{\perp}+R_{v} \ell_{z} v_{0}^{2}}$ reflect the impact velocity, the fluid properties and the orientation of SL.

\section{CONCLUSION}

Dynamics of random fractions flow of sliding contact collector elements destruction in steady-state was viewed. It was viewed for sliding interaction of collector pairs in space limited by area of sliding.

First it was considered the wear of sliding contact elements from gravity and orientation on space (deviation angle from the horizontal).

The model of thin layer in space limited by area of sliding contact collector elements was created. This model sets for research of fractions flow of sliding collector contact elements relation in steady state. Verification of this model was taken by numerical analysis. Contact area has the dynamically varying geometry of the sliding layer and changing form factions destruction.

\section{REFERENCES}

[1] H Kijima, "Influence of roll radius on roughness transfer in skin-pass rolling of steel strip", Journal of Materials Processing Technology, 214. Issue 5, pp. 1111-1119, 2014

[2] N.P. Suh. "The delamination theory of wear", Wear, 25(1), pp 111-124, 1973.

[3] J.F Archard, "Contact and rubbing of that surfaces", Journal of Applied Physics, 24(8). pp. 98!-988, 1953

[4] E Cerri, and P. Leo, "Mechanical properies evolution during postwelding-heat treatments of double-lap friction stir welded joints", Materials and Design, 32(6) pp 3465-3475, 2011

[5] R.S Mishra, and ZY Ma, "Friction stir welding and processing", Materals Science and Engineering R Reports, 50, Issue 1-2, pp 1-78, 2005
16] V.S Deeva. "Trajectory dispersion of sliding contact fractions", TUSUR Reports, 2(22), Part.1, pp. 249-254. 2010

[7] VS Deeva, and SM Slobodyan. "Action of isomorphic sliding currentcollect destruction", Energefic (2011), 9, pp. 36-38

18] VS. Deeva, and SM. Slobodyan. "Physical model of space of current collection interaction". The News of Altai State University, 1//(77), pp $157-161.2013$

[9] M.S. Slobodyan, and S.M. Slobodyan, "Model of electrical contac action", Equipment and systems Guidance, control, diagnostics, 2, pp 42-47, February 2010

[10] V S Deeva, M S Slobodyan, and S M Slobodyan, "Estimation of stochastic moving survivability of curtent collection". E-LECTRO Electrical engineering electricity, electrical industry, 6, pp 47-50, June 2010

[11] V.S. Deeva, S.M. Slobodyan, "Probabilistic estimation of the survivability of the lamellar current collection", Energetic, 10, pp 5356, October 2012

[12] M.S. Slobodyan, and S.M. Slobodyan, "Markơ model of great vitality by slippery contact of electrical machines", Testing Diagnostics (2011), 2, pp. 61-66

[13] V.S. Deeva, MS. Slobodyan, and S.M. Slobodyan, "Survivability of electrical machine brush contact", Electricity, 4, pp 45-49. April 2013

[14] V.S. Deeva, M.S. Slobodyar, and S.M. Slobodyan, "Wear of a sliding contact on the time in commutator", Testing Diagnostics, 6, pp. 59-65, June 2013.

[15] VS Deeva, and SM Slobodyan, "Dynamics of elecirical conduction field of the sliding current collector". Applied Mechanics and Materials, Vol 698 (2015) pp 258-263

[16] S.M. Slobodyan, V.I Kohanov, and S.A Shishigin, "Time trend of specual radiation from laser spark". In: 11-th International conference on spectral line shapes. Scientific program and abstract Carry le Rouet. June 8 12, 1992 pp A01-A02.

[17] S.M Slobodyan, "Television diagnosis laser beam" (2006), Bamaul Azbuka. 224 p. ISBN 5-93957-161-1

[18] A A Ponomarev, S.M Slobodyan "System" for automated control of laser-induced heasing of materials", Testing Diagnostics, 3, pp 62-63, Mars 2010

[19] V.F Volkov, A.K Peshel, SM Stobodyan, and IS Iyn'shkın "Registration of a Pulsed Laser Beam by a Matrix of Charge-coupled Devices", Instruments and Experimental Techniques, N-Y.24 (No 6 P1. 2), pp 1522-1524 (1981).

[20] V.A Arutyunov, and S.M Slobodyan, "Investigation of a CCD Wave Front Sensor of an Adaptive Optics Radiation Focusing Sjstem ${ }^{\text {rh }}$, Instruments and Experimental Techniques, N.-Y., 28, pp. 160-162 (1985).

[21] S.M. Slobodyan, "The Change in Fractal Dimensions When an Image is Degraded" // Measurement Techniques Springer US (2004), 47, No 8 pp. $28-34$

[22] S.M Slobodyan, "Estimation for fractal of signal/noise relations" // Measurement Techniques. Springer US (2008), 51, No 1, pp 5-8

[23] V.A. Arutyunov, V.G. Mel'nikov, S.M. Slobodyan, D.P. Chaporov, and ON Popov, "Sources of measurement error of fast-flowing process parameters by charge-coupled devices" // Measurement Techniques (1983), 26, pp 641-644, August 01, 1983

[24] V.I Bukaryi, NI Mishchenko, SM Slobodyan, D. Chaporov, "Measuring the Distribution of Radiation Intensiry in the Cross Section of a Laser Beam" // Instruments and experimental techniques (1976). New York, No 2, pp 166-168

[25] S.M Slobodyan, "Tracking optical phasometer", Bulletin of the Tomsk Polytechnic University (2003), 306, No. 6, pp. 101-106

[26] MS Slobodyan, SA Shishigin, and S.M Slobodyan, "Method of acousic sensor diagnosis" // Measurement Techniques Springer US (2008), 51, No 7, pp 798-801

[27] S M Slobodyan. "Optimizing phase-space scanning for a dynamic system monitoring chaotic media", Measurement Techniques Springer US (2006). 49. No 1,pp $1-6$ 